Original Research Paper

\title{
Birkhoff Orthogonality and Different Particular Cases of Carlsson's Orthogonality on Normed Linear Spaces
}

\author{
Prakash Muni Bajracharya and Bhuwan Prasad Ojha \\ Central Department of Mathematics, Kirtipur, Kathmandu, Nepal
}

\author{
Article history \\ Received: 21-02-2020 \\ Revised: $15-05-2020$ \\ Accepted: 21-07-2020 \\ Corresponding Author: \\ Bhuwan Prasad Ojha \\ Central Department of \\ Mathematics, Kirtipur, \\ Kathmandu, Nepal \\ Email: bhuwanp.pjha@gmail.com
}

\begin{abstract}
Let $x, y \in X$, where $X$ is an inner-product space. We say $x$ is orthogonal to $y$ if $\langle x, y\rangle=0$. When we move to general normed spaces there are many possibilities of extending the notion of orthogonality. Since 1934, different types of orthogonality relations in normed spaces have been introduced and studied. In this study, we enlist some properties of Birkhoff's orthogonality and Carlsson's orthogonality along with it we introduce two new particular cases of Carlsson's orthogonality and check some properties of othogonality in relation to these particular cases in normed spaces.
\end{abstract}

Keywords: Birkhoff Orthogonality, Carlsson Orthogonality, Minkowski Plane, Pythagorean Orthogonality, Robert Orthogonality

\section{Introduction}

Let $X^{*}$ be dual space of a normed space $X$ and $S_{X}$ denotes the unit sphere of $X$ given by $S_{X}=\{x \in X:\|x\|=$ 1\}. The Hermite-Hadamard's inequality defined in (Mihai et al., 2018) is given by:

$$
(b-a) f\left(\frac{a+b}{2}\right) \leq \int_{a}^{b} f(t) d t \leq(b-a) \frac{f(a)+f(b)}{2} .
$$

We briefly describe the content of this paper: Section 1 contains the review of various properties and characterizations of Birkhoff orthogonality in normed linear spaces. Section 2 contains Carlsson orthogonality in classical sense and in $\mathrm{HH}$-sense with the help of $\mathrm{p}-\mathrm{HH}$ norm. Section 3 includes the main results of our research by introducing new cases of Carlsson orthogonality verifying some properties of the inner-product space in relation of these orthogonalities.

There are different orthogonality relations studied in the general normed linear space since from 1934. The Robert orthogonality condition which was introduced by Robert in 1934 is known as the first orthogonality defined in general normed spaces (Birkhoff, 1935). Robert orthogonality implies both Birkhoff orthogonality introduced by (Birkhoff, 1935) and isosceles orthogonality introduced by (James, 1945). Generalizing the Isosceles, Robert and Pythagorean orthogonalities in the normed space, (Carlsson, 1962) introduced a new type of orthogonality. After that numerous notions of orthogonality have been introduced. Kikianty and Dragomir (2010) introduced $p$ - $H H$-norms $(1 \leq p<\infty)$ and some notions of orthogonality have been introduced by utilizing 2-HH-norm, which are closely related to the classical Pythagorean orthogonality and isosceles orthogonality. Kapoor and Prasad (1978) proved uniqueness property of isosceles orthogonality.

Mizuguchi (2017) let $(X,\|\cdot\|)$ be a real normed space. For any vector $x, y \in X$, we say that $x$ is orthogonal to $y$ $\left(x \perp_{B} y\right)$ in the sense of Birkhoff if:

$$
\|x\| \leq\|x+\lambda y\| \text { for all } \lambda \in \mathbb{R}
$$

James was the first who did a comprehensive study of the properties of Birkhoff orthogonality and therefore Birkhoff orthogonality is also known to as BirkhoffJames orthogonality. James (1945) introduced Isosceles and Pythagorean orthogonality proved that if for every $x$ it is possible to find a vector $y$ in a two dimensional subspace containing $x$ such that $x \perp_{B} y$; then the space is necessarily an inner product space (Dragomir and Kikianty, 2010). If $X$ is an inner product Space, then $\perp_{B}$ coincide with the standard orthogonality in inner product space. It is obvious that Birkhoff orthogonality is homogeneous; however, it is in general, neither symmetric nor additive.

\section{Definition (Martini and Spirova, 2010)}

A hyperplane of a normed linear space $X$ is any proper closed linear subset $H$ which is not properly contained in a proper linear subset of $X$, or any translation $x+H$ of such a linear subset $H$.

If $\left\{y_{n}\right\}$ is a sequence converging to $y, x$ is orthogonal to $\left\{y_{n}\right\}$, then $x \perp y$. Hence for any $x$, the set 
of all $y$ with $x \perp y$ is closed. for any element of a normed linear space there is at least one hyperplane $H$ through the origin with $x \perp H$.

\section{Properties of Birkhoff Orthogonality}

The following are the main properties of Birkhoff orthogonality (Alonso, 1997):

(i) Birkhoff-James orthogonality is not symmetric in general; however, if $\operatorname{dim} X \geq 3$ then it is symmetric if and only if $X$ is an inner product space. For $\operatorname{dim} X=2$ Birkhoff orthogonality is symmetric if and only if $X$ has a norm whose spheres are regular hexagon

(ii) It has the properiy of right and left-existence

(iii) It is right (left) unique if and only if the space is smooth (strictly convex)

(iv) It is always homogeneous

(v) Right additivity implies smoothness and conversely

(vi) It can always be extended by the right: If $x \perp_{B} y$, then there exist a closed hyperplane $P \subset X$ such that $y \in H$ and $x \perp_{B} H$

(vii) On the basis homogeneity, the Birkhoff-James orthogonality can always be extended by the left if $\operatorname{dim} X=2$ and in case of $\operatorname{dim} X \geq 3$, the property is a characteristic of inner product space and can be stated, equivalently, as follows:

$X$ is an inner product space if and only if for every $x \in X$ there exist a closed hyperplane $H \subset X$ such that $P \perp_{B}$ X

A real normed linear space of dimension $(\geq 3)$ is an inner product space if and only if B-orthogonality is symmetric.

\section{Characterization of Birkhoff Orthogonality}

Theorem 1.1 (Alonso, 1997)

Let $x, y \in X$, where $X$ is a normed space, then $x \perp_{B} \alpha x$ $+y, \Leftrightarrow X^{*}$ with $|f(x)|=\|f\|\|x\|$ where, $\alpha=-\frac{f(y)}{f(x)}$.

\section{Corollary 1.2}

Let $x, y \in X$, where $X$ is a normed space. Then $x \perp_{B} y$, $\Leftrightarrow \exists f \in X^{*} \backslash\{0\}:|f(x)|=\|f\|\|x\|$.

Definition (Semi-Inner Product, (Demalya et al., 2019))

A semi-inner product defined on a real normed space $X$ is a mapping of $X \times X$ into $R$ such that if for any $x, y \in$ $X$ there corresponds a real number $(x, y)$ such that the following properties hold:

(i) $(x+y, z)=(x, z)+(y, z)$ for all $x, y, z \in X$ (ii) $(\lambda x, y)=\lambda(x, y)$ for all $\lambda \in \mathbb{R}, x \in X$

(iii) $(x, x)>0$ for all $x \neq 0$

(iv) $(x, y)^{2} \leq(x, x)(y, y)$ for all $x, y \in X$

Every normed space $X$, where the norm on $X$ is defined by $\|x\|=\sqrt{(x, x)}$ is a semi-inner product space and norm of any normed linear space can be generated with the help of semi-inner product in infinitely many ways (Alonso, 1997). Birkhoff orthogonality can also be characterized with the help of semi-inner product. Giles (1967) added homogeneity in the second variable in the definition of semi- inner product, namely:

$$
(x, \lambda y)=\lambda(x, y) \text { for all } \lambda \in \mathbb{R}
$$

Let $x, y \in X$, where $X$ is a normed space and (.,.) be a semi inner product generating the norm of normed linear space $X$. Then $x$ is said to be orthogonal to $y$ in the sense of Lummer relative to semi inner product (.,.) if (.,.) $=0$ and written as $x \perp_{L} y$. Dragomir and Kikianty (2010) proved the following characterization of Birkhoff orthogonality.

\section{Theorem 1.3 (Alonso, 1997)}

Let $x, y \in X$, where $X$ is an inner-product space. Then $x \perp_{B} y$ iff $x \perp_{L} y$ relative to some semi inner product which generate the norm of $X$.

\section{Theorem 1.4 (Alonso, 1997)}

Let $x, y \in X$, where $X$ is an inner-product space. Then the following statements are equivalent:

(i) $x \perp_{B} y$

(ii) for every semi- inner product (.,.) generating the norm of $X$, there is $n$ inequality:

$$
(y, x+\xi y) \leq 0 \leq(y, x+\eta y) \text { for all } \xi<0<\eta .
$$

\section{Theorem 1.5. (Birkhoff, 1935)}

Let $X$ be a normed linear space. Then Birkhoff orthogonality is homogeneous in $X$.

\section{Definition}

A normed space $X$ is said to be strict convex (or rotund) if for every $x, y \in X$ with $\|x\|=\|y\|=1$ ) $x+y<2$, for $x \neq y$.

A normed space with such a norm is called a strictly convex normed space (or rotund space).

\section{Theorem 1.6. (Alonso, 1997)}

Let $X$ is normed space with $\operatorname{dim} X \geq 3$. Then $X$ is an inner product space iff Bifkhoff orthogonality is symmetric in $X$.

If Birkhoff orthogonality is symmetric in a strictly convex normed space $X$ with $\operatorname{dim} X \geq 3$. Then the space 
is an inner product space. Therefore the assumption on dimension of the space in relation to above theorem can not be omitted. To study about the symmetry of Birkhoff orthogonality, James provided an example of radon in the paper (James, 1945), on which the Birkhoff orthogonality is symmetric.

\section{Existence}

For the existence property of Birkhoff orthogonality we have the following result.

\section{Theorem 1.7 (Right Existence, (Alonso, 1997))}

Let $x, y \in X$, where $X$ is a normed space. Then there exist a real number $\xi$ such that $x \perp_{B} \xi x+y$ and such number $\xi$ satisfies $|\xi| \leq \frac{\|y\|}{\|x\|}$. If $x \perp_{B} \xi x+y$ and $x \perp_{B} \eta x+y$, then $x \perp_{B}$ $\gamma x+y$ holds for any real number between $\xi$ and $\eta$.

\section{Remark}

In the above theorem along with continuity of Birkhoff orthogonality imply that; for every any $x, y \in$ $X$, there exists a closed interval $[\xi, \eta]$ such that for each number $\alpha \in[\xi, \eta], x \perp_{B} \alpha x+y$ holds.

\section{Theorem 1.8 (Birkhoff, 1935)}

Let $x, y \in X$, where $X$ is a normed space with $x \neq 0$. If:

$$
\begin{aligned}
& \xi=\frac{1}{\|x\|}=\lim _{x \rightarrow \infty}(\|n x\|-\|n x+y\|) \\
& \eta=\frac{1}{\|x\|}=\lim _{x \rightarrow \infty}(\|n x-y\|-\|n x\|),
\end{aligned}
$$

then $\xi$ and $\eta$ are the smallest largest values of the scalar $\alpha$ such that $x \perp_{B}(\alpha x+y)$.

If $x \perp_{B}(\alpha x+y)$ and $y \perp_{B}(\bar{\alpha} y+x)$, the relationship between the scalars $\alpha$ and $\bar{\alpha}$ is given in the following theorem.

\section{Theorem 1.9 (James, 1945)}

Let $x, y \in X$, where $X$ is a normed space. If $x \perp_{B}(\xi x+$ $y)$ and $y \perp_{B}(\bar{\xi} y+x)$ then $|\xi \bar{\xi}| \leq 1$ and Birkhoff orthogonality is symmetric iff for any zero vectors $x, y$ and scalars $\xi, \bar{\xi}$ such that $x \perp_{B}(\xi x+y)$ and $y \perp_{B}(\bar{\xi} y+x)$, the inequality $\alpha \bar{\xi} \geq 0$ holds.

\section{Theorem 1.10 (Left Existence, (Alonso, 1997))}

Let $x, y \in X$, where $X$ is a normed space. $\exists \xi \in \mathbb{R}:(\xi x$ $+y) \perp_{B} x$ and $\|\xi x+y\|=\inf \{\|\eta x+y\|: \eta \in \mathbb{R}\}$. Moreover, if $\xi x+y \perp_{B} x$ and $\eta x+y \perp_{B} x$, then $\gamma x+y \perp_{B} x$ holds for any number between $\xi$ and $\eta$.

\section{Note}

For any closed hyperplane $H$ through origin, if $x \perp_{B} y$ $\left(y \perp_{B} x\right.$ respectively) holds for each $y \in H$, then we say that $x$ is Birkhoff orthogonal to $H$ and denoted by $x \perp H$ ( $H$ is Birkhoff orthogonal to $y, H \perp_{B} y$, resp).

\section{Theorem 1.11. (Spiorava and Martini, 2007)}

Let $x \in X$, where $X$ is a normed space. Then there exists a hyper-plane $H \subset X$ such that $x \perp_{B} H$.

\section{Theorem 1.12. (Spiorava and Martini, 2007)}

A Banach space $X$ is reflexive if and only if for any hyperplane $H \subset X$ there exist a vector $x \in X \backslash\{0\}$ such that $x \perp_{B} H$.

\section{Theorem 1.13. (Birkhoff, 1935)}

If the dimension of a normed space $X$ is greater or equal to 3 and $H$ be a hyperplane of $X$, then the following conditions are equivalent:

(i) $\forall H \subset X, \exists x \in X \backslash\{0\}: H \perp_{B} x$.

(ii) $\forall x \in X, \exists H \subset X: H \perp_{B} x$.

(iii) The normed space $X$ is an inner product space.

\section{Uniqueness}

For the uniqueness property of Birkhoff orthogonality Alonso (1997) proved the following results.

\section{Definition (Birkhoff, 1935)}

An orthogonality in the sense of Birkhoff-James is right-unique iff for no elements $x(\neq 0)$ and $\mathrm{y}$ there is more than one number $\alpha$ for which $x \perp \alpha x+y$ and leftunique iff for no elements $x(\neq 0)$ and $y$ there is more than one number $\xi$ for which $(\alpha x+y) \perp_{B} x$. Moreover, if the orthogonality is symmetric, then it is right unique iff it is left unique.

\section{Theorem 1.14. (Alonso, 1997)}

For any normed linear space $X$ in which Birkhoff orthogonality is unique on the left if and only if $X$ is strictly convex.

\section{Theorem 1.15 (Alonso, 1997)}

In any normed linear space $X$, the following statements are true:

(i) If the Birkhoff-James orthogonality is unique on the left in $X^{*}$, them it is unique on the right in $X$ and for reflexive normed space, the reciprocal result is also true

(ii) If the Birkhoff-James orthogonality is unique on the right in $X^{*}$, then it is unique on the left and if 
the space weekly compact, the reciprocal result is also true

It is obvious that every Minkowski space is reflexive and its unit sphere $S_{X}=\{x \in X:\|x\|=1\}$ is compact. In that case the uniqueness of Birkhoff orthogonality on the left (as well as on the right) in a Minkowski space is equivalent to the uniqueness of Birkhoff orthogonality on the right (as well as on left) in $X^{*}$ (Spiorava and Martini, 2007).

\section{Additivity}

To determine the additivity and uniqueness properties of Birkhoff orthogonality are closely related, (Alasno et el., 2012) use the above theorem and the following result.

Theorem 1.16 (Alonso et al., 2012)

For any normed linear space $X$, the Birkhoff-James orthogonality in $X$ is additive on the right if and only if $X$ is smooth.

Theorem 1.17 (Alonso, 1997)

Let $X$ be a normed linear space with $\operatorname{dim} X \geq 2$ :

(i) For $\operatorname{dim} X=2$, the Birkhoff-James orthogonality is additive on the left in $X$ iff $X$ is strictly convex.

(ii) For $\operatorname{dim} X_{-} 3$, the Birkhoff-James orthogonality is additive on the left in $X$ iff $X$ is an inner product space

\section{Theorem 1.18 (Alonso et al., 2012)}

Any normed space $X$ with $\operatorname{dim} X \geq 3$ is an inner product space if and only if Birkhoff orthogonality is l.a.b.

\section{Carlssion Orthogonality}

Carlsson (1962) gave the definition of orthogonality in a new pattern which is applicable in an arbitrary normed space as follows:

\section{Definition}

In a normed linear space $X$ :

$$
x \perp y \Leftrightarrow \sum_{k=1}^{m} a_{k}\left\|b_{k} x+c_{k} y\right\|^{2}=0
$$

where, $m \geq 2$ and $a_{k}, b_{k}, c_{k}$ are real numbers such that:

$$
\sum_{k=1}^{m} a_{k} b_{k} c_{k}=1, \sum_{k=1}^{m} a_{k} b_{k}^{2}=\sum_{k=1}^{m} a_{k} c_{k}^{2}=0
$$

The Pythagorean and Isosceles orthogonalities introduced by James in (Dragomir and Kikianty, 2010) are the particular cases of Carlssion orthogonality. The Carlssion orthogonality has the following properties: (i) It has the property of non-degeneracy, simplification and continuity

(ii) Carlsson orthogonality is symmetric in some cases (for instance, Pythagorean and isosceles orthogonalities) are not symmetric in other cases; however, the example $x \perp y(C)$ when $\|x+2 y\|=\| x$ $2 y \|$ shows that, it may not be symmetric in all cases

(iii) It is either homogeneous or additive to the left if and only if the underlying normed space is an inner product space

(iv) It is existent to the right and to the left

(v) With regards to uniqueness, Carlsson orthogonality is non-unique when the space is non-rotund; in particular, Pythagorean orthogonality is unique, but Isosceles orthogonality is unique iff the underlying normed space is strictly convex

Definition. (Dragomir and Kikianty, 2012)

A normed linear space $(X,\|\|$.$) is uniformly convex if,$ given $\epsilon>0$ there exists $\delta>0$ such that $\left\|\frac{x+y}{2}\right\|<1-\delta$, whenever $\|x-y\| \geq \epsilon$ and $x, y \in S_{X}$. The function:

$$
\delta(\epsilon)=\inf \left\{1-\left\|\frac{x+y}{2}\right\|: x, y \in S_{X},\|x-y\| \geq \epsilon\right\}
$$

is called the modulus of uniform convexity of the space $(X,\|\cdot\|)$.

\section{Carlsson Orthogonality in HH-Sense}

Dragomir and Kikianty (2012) introduced Carlsson type orthogonality in terms of the 2-HH-norm, which generalizes the previous definition of Carlsson's orthogonality.

Definition: $p-H H$ - norm $(1 \leq p<\infty)$ on $\mathbb{X}^{2}$

For any $x, y \in X$ :

$$
\|(x, y)\|_{p-H H}=\left(\int_{0}^{1}\|(1-t) x+t y\|^{p} d t\right)^{\frac{1}{p}}
$$

Some new notation of orthogonality have been introduced by using the 2-HH norm, which are closely related to Pythagorean and Isosceles orthogonality. The results are summarized in (Dragomir and Kikianty, 2012) as follows:

Let $X$ be a normed space:

(i) A vector $x \in X$ is HH-P-orthogonal to $y \in X$ ( denoted by $x \perp_{H H-P}$ ) if and only if:

$$
\int_{0}^{1}\|(1-t) x+t y\|^{2} d t=\frac{1}{3}\left(\|x\|^{2}+\|y\|^{2}\right)
$$


(ii) A vector $x \in X$ is $H H$-I-orthogonal to $y \in X$ (denoted by $x \perp_{H H-I} y$ ) iff:

$$
\int_{0}^{1}\|(1-t) x+t y\|^{2} d t=\int_{0}^{1}\|(1-t) x-t y\|^{2} d t
$$

(iii) The homogeneity (or additivity) of the HH-P(and $\mathrm{HH}-\mathrm{I}$ ) orthogonality characterizes inner product space (Dragomir and Kikianty, 2012)

Definition. HH-C-Orthogonality (Dragomir and Kikianty, 2012)

Let $x$ and y be two vectors in $X$ and $t \in[0,1]$. Suppose that $(1-t) x \perp t y(C)$ almost everywhere on $[0,1]$ i.e:

$$
\sum_{k=1}^{m} \alpha_{i}\left\|\beta_{i} x+\gamma_{i} y\right\|^{2}=0
$$

for some $m, n \in N$ and real numbers $\alpha_{i}, \beta_{i}, \gamma_{i}$ such that:

$$
\sum_{k=1}^{m} \alpha_{i} \beta_{i} \gamma_{i}=1 \text { and } \sum_{k=1}^{m} \alpha_{i} \beta_{i}^{2}=\sum_{k=1}^{m} \alpha_{i} \gamma_{i}^{2}=0
$$

Then:

$$
\sum_{k=1}^{m} \alpha_{i} \int_{0}^{1}\left\|(1-t) \beta_{i} x+t \gamma_{i} y\right\|^{2} d t=0
$$

A normed space $(X,\|\|),. x \in X$ is said to be HH-Corthogonal to $y \in X$ (we denote it by $x \perp_{H H-C} y$ if and only if $x$ and $y$ satisfies (2) with conditions (1).

It can be shown that the HH-C- orthogonality is equivalent to the usual orthogonality in any inner product space. HH-P-orthogonality is a particular case of HH-Corthogonality which is obtained by choosing $m=3, \alpha_{1}=$ $1, \alpha_{2}=\alpha_{3}=1, \beta_{1}=\beta_{2}=1, \beta_{3}=0, \gamma_{1}=\gamma_{3}=1$ and $\gamma_{2}=0$.

Similarly, HH-I-orthogonality is also a particular case of $\mathrm{HH}-\mathrm{C}$-orthogonality. The following propositions follows by the definition of $\mathrm{Hh}$-C-orthogonality; and we omit the proof.

\section{Theorem 2.1. (Dragomir and Kikianty, 2012)}

HH-C-orthogolality satisfies the non-degeneracy, simplification and continuity.

With regard to symmetry, $\mathrm{HH}-\mathrm{C}$-orthogonality is symmetric in some cases, for example, HH-P- and HH-I-orthogonalities are symmetric (Dragomir and Kikianty, 2012).

\section{Example 1. (Dragomir and Kikianty, 2012)}

HH-C-orthogonality is not symmetric.

\section{Proof}

Define $x \perp_{H H-C_{2}} y$ to be:

$$
\int_{0}^{1}\|(1-t) x+2 t y\|^{2} d t=\int_{0}^{1}\|(1-t) x-2 t y\|^{2} d t .
$$

In $\mathbb{R}^{2}$ with $l^{1}$-norm, $x=(2 ; 1)$ is $H H$ - $C_{2}$-orthogonal to $y=\left(\frac{1}{2},-1\right)$ but $y \perp_{H H-C_{2}} x$.

Therefore we may conclude that HH-C-orthogonality is neither additive nor homogeneous depending on the fact that HH-P- and HH-I-orthogonalities are neither additive nor homogeneous (Dragomir and Kikianty, 2012).

\section{Main Result}

Roberts (1934), Pythagorean and Isosceles orthogonalities have been generalized by Carlsson in 1962 (Dragomir and Kikianty, 2012). These orthogonalities are obtained by assigning particular values of constants in a generalized Carlsson's orthogonality. In this section we will show how isosceles, Robert and Pyhagorean orthogonalities can be derived from the Carlsson orthogonality and obtain two new orthogonality relations for the Carlsson orthogonality.

\section{Isosceles Orthogonality}

A vector $x$ in $X$ is isosceles orthogonal the vector $y$ in $X$ if and only if $\|x+y\|=\|x-y\|$.

Proof

We have:

$$
\begin{aligned}
& \sum_{k=1}^{2} a_{k}\left\|b_{k} x+c_{k} y\right\|^{2}=0 \\
& \Rightarrow a_{1}\left\|b_{1} x+c_{1} y\right\|^{2}+a_{2}\left\|b_{2} x+c_{2} y\right\|^{2}=0 .
\end{aligned}
$$

Put $a_{1}=\frac{1}{2}, a_{2}=\frac{-1}{2}, b_{1}=b_{2}=1, c_{1}=1$ and $c_{2}=-1$.

Then:

$$
\begin{aligned}
& 0=a_{1}\left\|b_{1} x+c_{1} y\right\|^{2}+a_{2}\left\|b_{2} x+c_{2} y\right\|^{2} \\
& =\frac{1}{2}\|x+y\|^{2}+\frac{-1}{2}\|x-y\|^{2} . \\
& \Rightarrow\|x+y\|^{2}=\|x-y\|^{2} . \\
& \therefore\|x+y\|=\|x-y\| .
\end{aligned}
$$

Now:

$$
\begin{aligned}
& \sum_{k=1}^{2} a_{k} b_{k} c_{k}=a_{1} b_{1} c_{1}+a_{2} b_{2} c_{2}=\frac{1}{2} \times 1 \times 1+\frac{-1}{2} \times 1 \times-1=1 . \\
& \sum_{k=1}^{2} a_{k} b_{k}^{2}=a_{1} b_{1}^{2}+a_{2} b_{2}^{2}=\frac{1}{2} \times 1+\frac{-1}{2} \times 1=0 . \\
& \sum_{k=1}^{2} a_{k} c_{k}^{2}=a_{1} c_{1}^{2}+a_{2} c_{2}^{2}=\frac{1}{2} \times 1+\frac{-1}{2} \times 1=0 .
\end{aligned}
$$




\section{Robert Orthogonality}

A vector $x \in X$ is orthogonal to the vector $y \in X$ if and only if $\|x+\lambda y\|=\|x-\lambda y\|$ for all real $\lambda$.

\section{Proof}

We have:

$$
a_{1}\left\|b_{1} x+c_{1} y\right\|^{2}+a_{2}\left\|b_{2} x+c_{2} y\right\|^{2}=0 .
$$

Put $a_{1}=\frac{1}{2}, a_{2}=\frac{-1}{2}, b_{1}=b_{2}=1, c_{1}=\lambda$ and $c_{2}=-\lambda$. Then:

$$
\begin{aligned}
& 0=a_{1}\left\|b_{1} x+c_{1} y\right\|^{2}+a_{2}\left\|b_{2} x+c_{2} y\right\|^{2} \\
& =\frac{1}{2}\|x+\lambda y\|^{2}+\frac{-1}{2}\|x-\lambda y\|^{2} \\
& \Rightarrow\|x+\lambda y\|^{2}=\|x-\lambda y\|^{2} . \\
& \therefore\|x+\lambda y\|=\|x-\lambda y\| .
\end{aligned}
$$

Now:

$$
\begin{aligned}
& \sum_{k=1}^{2} a_{k} b_{k} c_{k}=a_{1} b_{1} c_{1}+a_{2} b_{2} c_{2}=\frac{1}{2} \times 1 \times \lambda+\frac{-1}{2} \times 1 \times-\lambda=\lambda \neq 0 . \\
& \sum_{k=1}^{2} a_{k} b_{k}^{2}=a_{1} b_{1}^{2}+a_{2} b_{2}^{2}=\frac{1}{2} \times 1+\frac{-1}{2} \times 1=0 . \\
& \sum_{k=1}^{2} a_{k} c_{k}^{2}=a_{1} c_{1}^{2}+a_{2} c_{2}^{2}=\frac{1}{2} \times \lambda^{2}+\frac{-1}{2} \times \lambda^{2}=0 .
\end{aligned}
$$

\section{Pythagorean Orthogonality}

A vector $x$ in $X$ is Pythagorean orthogonal the vector $y$ in $X$ if and only if:

$$
\|x-y\|^{2}=\|x\|^{2}+\|y\|^{2} .
$$

\section{Proof}

We have:

$$
\sum_{k=1}^{3} a_{k}\left\|b_{k} x+c_{k} y\right\|^{2}=0
$$$$
\Rightarrow a_{1}\left\|b_{1} x+c_{1} y\right\|^{2}+a_{2}\left\|b_{2} x+c_{2} y\right\|^{2}+a_{3}\left\|b_{3} x+c_{3} y\right\|^{2}=0 .
$$

Put $a_{1}=-1, a_{2}=a_{3}=1, b_{1}=b_{2}=1, b_{3}=0$ and $c_{1}=-1$, $c_{2}=0, c_{3}=1$. Then:

$$
\begin{aligned}
& 0=a_{1}\left\|b_{1} x+c_{1} y\right\|^{2}+a_{2}\left\|b_{2} x+c_{2} y\right\|^{2}+a_{3}\left\|b_{3} x+c_{3} y\right\|^{2} \\
& =(-1)\|x-y\|^{2}+\|x\|^{2}+\|y\|^{2} \\
& \Rightarrow\|x-y\|^{2}=\|x\|^{2}+\|y\|^{2} .
\end{aligned}
$$

Now:

$$
\sum_{k=1}^{3} a_{k} b_{k} c_{k}=1, \sum_{k=1}^{3} a_{k} b_{k}^{2}=0, \sum_{k=1}^{3} a_{k} c_{k}^{2}=0 .
$$

\section{Particular Case of Carlsson Orthogonality}

A vector $x$ in $X$ is orthogonal the vector $y$ in $X$ if and only if:

$$
2\|x+y\|^{2}+i\|x+i y\|^{2}=2\|x-y\|^{2}+i\|x-i y\|^{2} .
$$

\section{Proof}

We have:

$$
\begin{aligned}
& \sum_{k=1}^{4} a_{k}\left\|b_{k} x+c_{k} y\right\|^{2}=0 \\
& \Rightarrow a_{1}\left\|b_{1} x+c_{1} y\right\|^{2}+a_{2}\left\|b_{2} x+c_{2} y\right\|^{2} \\
& +a_{3}\left\|b_{3} x+c_{3} y\right\|^{2}+a_{4}\left\|b_{4} x+c_{4} y\right\|^{2}=0 .
\end{aligned}
$$

Put $a_{1}=b_{1}=c_{1}=1, a_{2}=\frac{i}{2}, b_{2}=1, c_{2}=i, a_{3}=-1, b_{3}$ $=1, c_{3}=-1, a_{4}=\frac{-i}{2}, b_{4}=1$ and $c_{4}=-i$. Then:

$$
\begin{aligned}
& 0=a_{1}\left\|b_{1} x+c_{1} y\right\|^{2}+a_{2}\left\|b_{2} x+c_{2} y\right\|^{2} \\
& +a_{3}\left\|b_{3} x+c_{3} y\right\|^{2}+a_{4}\left\|b_{4} x+c_{4} y\right\|^{2} \\
& =\|x+y\|^{2}+\frac{i}{2}\|x+i y\|^{2}+(-1)\|x-y\|^{2}-\frac{i}{2}\|x-i y\|^{2} \\
& =\|x+y\|^{2}-\|x-y\|^{2}+\frac{i}{2}=\left[\|x+i y\|^{2}+\|x-i y\|^{2}\right] . \\
& \therefore 2\|x+y\|^{2}+i\|x+i y\|^{2}=2\|x-y\|^{2}+i\|x-i y\|^{2} .
\end{aligned}
$$

Now:

$$
\begin{aligned}
& \sum_{k=1}^{4} a_{k} b_{k} c_{k}=a_{1} b_{1} c_{1}+a_{2} b_{2} c_{2}+a_{3} b_{3} c_{3}+a_{4} b_{4} c_{4} \\
& =1 \times 1 \times 1+\frac{i}{2} \times 1 \times i+-1 \times 1 \times-1+\frac{-i}{2} \times 1 \times-i \\
& \sum_{k=1}^{4} a_{k} b_{k}^{2}=a_{1} b_{1}^{2}+a_{2} b_{2}^{2}+a_{3} b_{3}^{2}+a_{4} b_{4}^{2} \\
& =1 \times 1+\frac{i}{2} \times 1+-1 \times 1+\frac{-i}{2} \times 1=0 . \\
& \sum_{k=1}^{4} a_{k} c_{k}^{2}=a_{1} c_{1}^{2}+a_{2} c_{2}^{2}+a_{3} c_{3}^{2}+a_{4} c_{4}^{2} \\
& =1 \times 1+\frac{i}{2} \times i^{2}+-1 \times 1+\frac{-i}{2} \times-i^{2}=0 .
\end{aligned}
$$

\section{Particular Case of Carlsson's Orthogonality}

A vector $x$ in $X$ is orthogonal the vector $y$ in $X$ if and only if: 


$$
\left\|x+\frac{1}{2} y\right\|^{2}+\left\|x-\frac{1}{2} y\right\|^{2}=\frac{1}{2}\|\sqrt{2} x+y\|^{2}+\|x\|^{2} .
$$

\section{Proof}

We have:

$$
\begin{aligned}
& \sum_{k=1}^{4} a_{k}\left\|b_{k} x+c_{k} y\right\|^{2}=0 \\
& \Rightarrow a_{1}\left\|b_{1} x+c_{1} y\right\|^{2}+a_{2}\left\|b_{2} x+c_{2} y\right\|^{2} \\
& +a_{3}\left\|b_{3} x+c_{3} y\right\|^{2}+a_{4}\left\|b_{4} x+c_{4} y\right\|^{2}=0 .
\end{aligned}
$$

Put $a_{1}=a_{2}=1, a_{3}=\frac{-1}{2}, a_{4}=\frac{-1}{2}, b_{1}=1, b_{2}=1, b_{3}$ $=\sqrt{2}, b_{4}=\sqrt{2}, c_{1}=\frac{1}{2}, c_{2}=\frac{-1}{2}, c_{3}=1$ and $c_{4}=0$. Then:

$$
\begin{aligned}
& 0=a_{1}\left\|b_{1} x+c_{1} y\right\|^{2}+a_{2}\left\|b_{2} x+c_{2} y\right\|^{2} \\
& +a_{3}\left\|b_{3} x+c_{3} y\right\|^{2}+a_{4}\left\|b_{4} x+c_{4} y\right\|^{2} \\
& =\left\|x+\frac{1}{2} y\right\|^{2}+\left\|x-\frac{1}{2} y\right\|^{2}-\frac{1}{2}\|\sqrt{2} x+y\|^{2}-\frac{1}{2}\|\sqrt{2} x\|^{2} \\
& \therefore\left\|x+\frac{1}{2} y\right\|^{2}+\left\|x-\frac{1}{2} y\right\|^{2}=\frac{1}{2}\|\sqrt{2} x+y\|^{2}+\|x\|^{2} .
\end{aligned}
$$

Now:

$$
\begin{aligned}
& \sum_{k=1}^{4} a_{k} b_{k} c_{k}=a_{1} b_{1} c_{1}+a_{2} b_{2} c_{2}+a_{3} b_{3} c_{3}+a_{4} b_{4} c_{4} \\
& =1 \times 1 \times \frac{1}{2}+1 \times 1 \times \frac{-1}{2}+\frac{-1}{2} \times \sqrt{2} \times 1+\frac{-1}{2} \times \sqrt{2} \times 0=\frac{-1}{\sqrt{2}} . \\
& \sum_{k=1}^{4} a_{k} b_{k}^{2}=a_{1} b_{1}^{2}+a_{2} b_{2}^{2}+a_{3} b_{3}^{2}+a_{4} b_{4}^{2} \\
& =1 \times 1+1 \times 1+\frac{-1}{2} \times 2+\frac{-1}{2} \times 2=0 . \\
& \sum_{k=1}^{4} a_{k} c_{k}^{2}=a_{1} c_{1}^{2}+a_{2} c_{2}^{2}+a_{3} c_{3}^{2}+a_{4} c_{4}^{2} \\
& =1 \times \frac{1}{4}+1 \times \frac{1}{4}+\frac{-1}{2} \times 1+\frac{-1}{2} \times 0=0 .
\end{aligned}
$$

\section{Properties of Orthogonality Relation of Type (I)}

Orthogonal relation $\|x+y\|^{2}+i\|x+i y\|^{2}=2\|x-y\|^{2}+$ $i \quad\|x-i y\|^{2}$ satisfies non-degeneracy, simplification and continuity:

(i) Non-degeneracy: $x \perp x \Rightarrow x=0$.

Proof

We have:

$$
2\|x+y\|^{2}+i\|x+i y\|^{2}=2\|x-y\|^{2}+i\|x-i y\|^{2} .
$$

If $x \perp x$, then:

$$
\begin{aligned}
& 2\|2 x\|^{2}+i\|x(1+i)\|^{2}=2\|x-x\|^{2}+i\|x(1-i)\|^{2} \\
& \Rightarrow 8\|x\|^{2}+i(1+i)(1-i)\|x\|^{2}=i(1-i)(1+i)\|x\|^{2} \\
& \Rightarrow 8\|x\|^{2}=0 \\
& \Rightarrow x=0 .
\end{aligned}
$$

(ii) Simplification: If $x \perp y \Rightarrow \lambda x \perp \lambda y$.

Proof

We have:

$$
\begin{aligned}
& 2\|x+y\|^{2}+i\|x(x+i y)\|^{2}=2\|x-y\|^{2}+i\|x-i y\|^{2} \\
& \Rightarrow 2|\lambda|^{2}\|x+y\|^{2}+i|\lambda|^{2}\|x+i y\|^{2} \\
& =2|\lambda|^{2}\|x-y\|^{2}+i|\lambda|^{2}\|x-i y\|^{2} \\
& \Rightarrow 2\|\lambda x+\lambda y\|^{2}+i\|\lambda x+i \lambda y\|^{2} \\
& =2\|\lambda x-\lambda y\|^{2}+i\|\lambda x-i \lambda y\|^{2} \\
& \Rightarrow \lambda x \perp \lambda y .
\end{aligned}
$$

(iii) Continuity: If $\left(x_{n}\right),\left(y_{n}\right) \subset X$, such that $x_{n} \perp y_{n}$ for every $n \in 2 \mathbb{N}, x_{n} \rightarrow x, y_{n} \rightarrow y$, then $x \perp y$.

\section{Proof}

Let $\left(x_{n}\right),\left(y_{n}\right) \subset X$, such that $x_{n} \perp y_{n}$. Then:

$$
\begin{aligned}
& 2\left\|x_{n}+y_{n}\right\|^{2}+i\left\|x_{n}+i y_{n}\right\|^{2}=2\left\|x_{n}-y_{n}\right\|^{2}+i\left\|x_{n}-i y_{n}\right\|^{2} \\
& \Rightarrow 2\|x+y\|^{2}+i\|x+i y\|^{2}=2\|x-y\|^{2} \\
& +i\|x-i y\|^{2}\left(\therefore x_{n} \Rightarrow x, y_{n} \rightarrow y\right) \\
& \Rightarrow x \perp y .
\end{aligned}
$$

\section{Properties of Orthogonality Relation of Type (II)}

The orthogonality relation:

$$
\left\|x+\frac{1}{2} y\right\|^{2}+\left\|x-\frac{1}{2} y\right\|^{2}=\frac{1}{2}\|\sqrt{2} x+y\|^{2}+\|x\|^{2}
$$

satisfies non-degeneracy, simplification and continuity:

(i) Non-degeneracy: $x \perp x \Rightarrow x=0$.

Proof

If $x \perp x$, then by definition we have:

$$
\begin{aligned}
& \left\|x+\frac{1}{2} x\right\|^{2}+\left\|x-\frac{1}{2} x\right\|^{2}=\frac{1}{2}\|\sqrt{2} x+x\|^{2}+\|x\|^{2} \\
& \Rightarrow\|x\|=0 \\
& \Rightarrow x=0 .
\end{aligned}
$$


(ii) Simplification: $x \perp y \Rightarrow \lambda x \perp \lambda y$.

\section{Proof}

If $x \perp y$, then by definition we have:

$$
\left\|x+\frac{1}{2} y\right\|^{2}+\left\|x-\frac{1}{2} y\right\|^{2}=\frac{1}{2}\|\sqrt{2} x+y\|^{2}+\|x\|^{2} .
$$

If $\lambda \in \mathbb{R}$, then:

$$
\begin{aligned}
& |\lambda|^{2}\left\|x+\frac{1}{2} y\right\|^{2}+|\lambda|^{2}\left\|x-\frac{1}{2} y\right\|^{2}=\frac{1}{2}|\lambda|^{2}\|\sqrt{2} x+y\|^{2}+|\lambda|^{2}\|x\|^{2} \\
& \Rightarrow\left\|\lambda x+\frac{1}{2} \lambda y\right\|^{2}+\left\|\lambda x-\frac{1}{2} \lambda y\right\|^{2} \\
& =\frac{1}{2}\|\sqrt{2} \lambda x+\lambda y\|^{2}+\|\lambda x\|^{2} \\
& \Rightarrow \lambda x \perp \lambda y .
\end{aligned}
$$

(iii) Continuity: If $\left(x_{n}\right),\left(y_{n}\right) \subset X$, such that $x_{n} \perp y_{n}$ for every $n \in \mathbb{N}, x_{n} \rightarrow x, y_{n} \rightarrow y$, then $x \perp y$.

\section{Proof}

Let $\left(x_{n}\right) ;\left(y_{n}\right) \subset X$, such that $x_{n} \perp y_{n}$ for all $n$. Then:

$$
\begin{aligned}
& \forall n\left\|x_{n}+\frac{1}{2} y_{n}\right\|^{2}+\left\|x_{n}-\frac{1}{2} y_{n}\right\|^{2}=\frac{1}{2}\left\|\sqrt{2} x_{n}+y_{n}\right\|^{2}+\left\|x_{n}\right\|^{2} \\
& \Rightarrow\left\|x+\frac{1}{2} y\right\|^{2}+\left\|x-\frac{1}{2} y\right\|^{2}=\frac{1}{2}\|\sqrt{2} x+y\|^{2} \\
& +\|x\|^{2}\left(\therefore x_{n} \rightarrow x, y_{n} \rightarrow y\right) \\
& \Rightarrow x \perp y .
\end{aligned}
$$

(iv) Homogeneity: If $x \perp y$, then $\lambda x \perp \lambda y$ holds for any real number $\lambda, \mu \in \mathbb{R}$.

\section{Proof}

If $x \perp y$, then we have:

$$
\left\|x+\frac{1}{2} y\right\|^{2}+\left\|x-\frac{1}{2} y\right\|^{2}=\frac{1}{2}\|\sqrt{2} x+y\|^{2}+\|x\|^{2} .
$$

Now:

$$
\begin{aligned}
& \left\|\lambda x+\frac{1}{2} \mu y\right\|^{2}+\left\|\lambda x-\frac{1}{2} \mu y\right\|^{2}-\frac{1}{2}\|\sqrt{2} \lambda x+\mu y\|^{2}-\|\lambda x\|^{2}=0 \\
& \Leftrightarrow\left\langle\lambda x+\frac{1}{2} \mu y, \lambda x+\frac{1}{2} \mu y\right\rangle+\left\langle\lambda x-\frac{1}{2} \mu y, \lambda x-\frac{1}{2} \mu y\right\rangle \\
& -\frac{1}{2}\langle\sqrt{2} \lambda x+\mu y, \sqrt{2} \lambda x+\mu y\rangle-\langle\lambda x, \lambda x\rangle=0 \\
& \Leftrightarrow-2 \sqrt{2} \lambda \mu\langle x, y\rangle=0 .
\end{aligned}
$$

Therefore the given orthogonality is homogeneous if and only if the space is an inner product space.

\section{Conclusion}

Carlsson (1962) introduced a more general type of orthogonality and also proved that Isosceles, Robert and Pythagorean orthogonalities are special cases. The most interesting thing here is that all three particular cases may not have common properties of inner-product space. There are some common properties like non-degeneracy, simplification and continuity, but some properties may be different in different particular cases. For instance, Robert orthogonality is homogeneous and symmetric. On the other hand (Alonso, 1997), Isosceles and Pythagorean orthogonalities are either homogeneous or additive if and only if the norm is induced by an inner-product. In this article we introduced two new special case of generalized Carlsson's orthogonality namely of type (I) and type (II) and we check some properties of inner-product spaces in relation to these orthogonalities. We proved that orthogonality relation of type (I) satisfies non-degeneracy, simplification and continuity; however, the orthogonality relation of type (II) satisfies these three properties as well as homogeneity iff the norm is induced by an inner-product.

\section{Acknowledgmen}

I would like to express my gratefulness to my $\mathrm{PhD}$ supervisor for his encouragement and support on this paper. I also would like to express my deep gratitude to the Apex College. Finally, the authors are highly grateful to the editor and referees for their valuable suggestions and comments.

\section{Author's Contribution}

Both of the authors contributed equally on the article.

\section{Ethic}

This work has not been published or submitted for publication to any journals. All the works consulted have been properly cited and mentioned in the reference section. There is no any ethical issue regarding the publication of the article.

\section{References}

Alonso, J. (1997). Some properties of Birkhoff and Isosceles orthogonality in normed linear spaces. Bull. Un. Math. Ital.

Alonso, J., Martini, H., \& Wu, S. (2012). On Birkhoff orthogonality and isosceles orthogonality in normed linear spaces. Aequationes mathematicae, 83(1-2), 153-189. 
Birkhoff, G. (1935). Orthogonality in linear metric spaces. Duke Mathematical Journal, 1(2), 169-172.

Carlsson, S. O. (1962). Orthogonality in normed linear spaces. Arkiv för Matematik, 4(4), 297-318.

Demalya, S., Mal, A., \& Paul, K. (2019). Some remarks on Birkhoff-James orthogonality of linear operators. Expositiones Mathematicae, 38(1), 138-147.

Dragomir, S. S., \& Kikianty, E. (2010). Orthogonality connected with integral means and characterizations of inner product spaces. Journal of Geometry, 98(1-2), 33-49.

Dragomir, S. S., \& Kikianty, E. (2012). On Carlsson type orthogonality and characterization of inner product spaces. Filomat, 26(4), 859-870.

Giles, J. R. (1967). Classes of semi-inner-product spaces. Transactions of the American Mathematical Society, 129(3), 436-446.

James, R. C. (1945). Orthogonality in normed linear spaces. Duke Mathematical Journal, 12(2), 291-302.

Kapoor, O. P., \& Prasad, J. (1978). Orthogonality and characterizations of inner product spaces. Bulletin of the Australian mathematical Society, 19(3), 403-416.
Kikianty, E., \& Dragomir, S. S. (2010). HermiteHadmard's Inequality and the p-HH-norm on the cartesian product of two copies of a normed space. Math. Inequalities Applic.

Martini, H., \& Spirova, M. (2010). A new type of orthogonality for normed planes. Czechoslovak mathematical journal, 60(2), 339-349.

Mihai, M. V., Awan, M. U., Noor, M. A., Kim, J. K., \& Noor, K. I. (2018). Hermite-Hadamard inequalities and their applications. Journal of inequalities and applications, 2018(1), 1-9.

Mizuguchi, H. (2017). The differences between Birkhoff and isosceles orthogonalities in Radon planes. extracta mathematicae, 32(2), 173-208.

Roberts, B. D. (1934). On the geometry of abstract vector spaces. Tohoku Mathematical Journal, First Series, 39, 42-59.

Spiorava, M., \& Martini, H. (2007). The Feuerbach circle and orthocentricity in normed planes. ENSEIGNEMENT MATHEMATIQUE, 53(3-4), $237-258$ 